\title{
The impact of direct oral anti-coagulants on hip fracture management : a systematic review and meta-analysis
}

\author{
Samuel Holmes, Sarmad BAHNAM
}

From the Armidale Hospital, Armidale, NSW, 2350 Australia

Hip fractures are a common presentation in the elderly, a group who commonly have co-morbidities requiring the use of anticoagulants. Recently, direct oral anti-coagulants (DOAC) have become a popular method of anticoagulating patients. The primary aim of this review is to determine if the use of DOAC in elderly patients with hip fractures results in delays to theatre and/or an increased mortality risk.

Major databases including Pubmed, MEDLINE and Embase were searched for relevant studies and the included studies reference lists were screened for further studies. A total of $\mathbf{6}$ studies were included in meta-analysis which was undertaken using RevMan software with $95 \%$ confidence interval (CI). Compared to control groups, patients who were anticoagulated with DOAC and required operative management of a hip fracture experienced a statistically significant delay to treatment. However independent analysis showed these patients did not experience an increased mortality risk when compared to the control group. While data is limited, treating clinicians should be comforted by these findings as operative management can be safely delayed to reduce the risk of intraoperative bleeding.

Keywords : anti-coagulation ; DOAC ; NOAC ; hip ; fracture ; NOF ; neck of femur.

\section{INTRODUCTION}

The introduction of Direct or Novel Oral AntiCoagulants (DOAC) marked a revolution in medi- cal management by providing an alternative to Vitamin $\mathrm{K}$ antagonists which are limited by their narrow therapeutic window, variable dose-response, plethora of drug interactions and dose monitoring requirements (1). These benefits are offset in the acute emergency setting where decision making is complicated by difficulties in measuring drug concentrations and in reversing the anticoagulant $(2,3)$. Hip fractures remain a common trauma presentation in older populations and an increasing number of these patients are presenting while anticoagulated by DOAC (4-10). As the prevalence of these presentations increases (7), treating surgeons will be increasingly expected to consider the risks and benefits of management with limited research and many articles limited by sample sizes. In light of this, a systematic review and meta-analysis is most useful in determining generalizations for this population $(11,12)$; thus, this article will assess the impact of DOAC on hip fracture management by

Samuel Holmes ${ }^{1}$,

Sarmad Bahnam ${ }^{2}$

${ }^{1}$ Affiliation: Sydney Local Health District; NSW Health, NSW, Australia.

${ }^{2}$ Hunter New England Health District, NSW Health, NSW Australia.

Correspondence : Samuel Holmes, Sydney Local Health District, NSW Health, 19/2-6 Brown St Newtown, NSW, Australia, 2042,

Email: sholmes.ot@gmail.com

- 2021, Acta Orthopædica Belgica. 
assessing : 1, do these patients experience a delay to operative management compared to their nonanticoagulated peers and 2. do they assume a higher mortality risk.

\section{METHODS}

The databases Pubmed, MEDLINE and Embase were searched in August 2019 with no date exclusion for journals which compared perioperative outcomes of DOAC-using patients undergoing operative management of hip fractures compared with nonanticoagulated controls. The search strategy develop was : "Direct Oral anticoagulants" OR "New Oral anticoagulants" Or "Novel Oral anticoagulants" AND "femur" OR "Hip" AND "Fracture" with the only search limitation being 'human species'. The bibliographies of the included studies were screened for articles not discovered by this search strategy. Articles were included if they satisfied the following inclusion criteria: (i) comparing DOAC vs. Control, (ii) population required acute management of a hip fracture/s.

The data was extracted by the author (S.H) and cross checked by the author (S.B). Two primary outcomes were decided upon: (i) time to surgery, as this is an important consideration during the acute management of hip fractures, and (ii) all-cause mortality, as this forms a basis to determine the efficacy of treatment and has high homogeneity amongst studies. Secondary outcomes were determined by the availability of data while satisfying the studies definition. This included perioperative transfusion rate and deep vein thrombosis prevalence. The articles included in meta-analysis combined data from a variety of surgical techniques. This was not differentiated during meta-analysis as surgical technique was not relevant the aims of this research which is assess how DOAC use impacted time to surgery and mortality.

The meta-analysis was undertaken using RevMan software with $95 \%$ confidence interval (CI). Mean and standard deviation (SD) were used for the metaanalysis of continuous data, specifically Time-ToSurgery. Some included studies provided median and interquartile range (IQR) instead, so this data was converted to mean and SD. DOAC users are compared to a non-anticoagulated control group rather than vitamin $\mathrm{K}$ antagonist users for two reasons : (i) the availability of data and (ii) a control group theoretically has fewer comorbidities which reduces the number of confounders.

\section{RESULTS}

The search strategy resulted in a total of 65 articles (Figure 1) and after removing duplicates, 46 articles were analysed by the literature review. Articles were excluded if they did not provide quantitative data, lacked a control group, or failed to answer the research question. 29 articles were excluded from abstract and 17 articles were short listed for full text analysis. 7 were successful and the reference lists of these 7 articles were reviewed for other potential studies. A total of 13 potential articles were found, assessed and one was included in the meta-analysis. A total of 8 articles fitted the
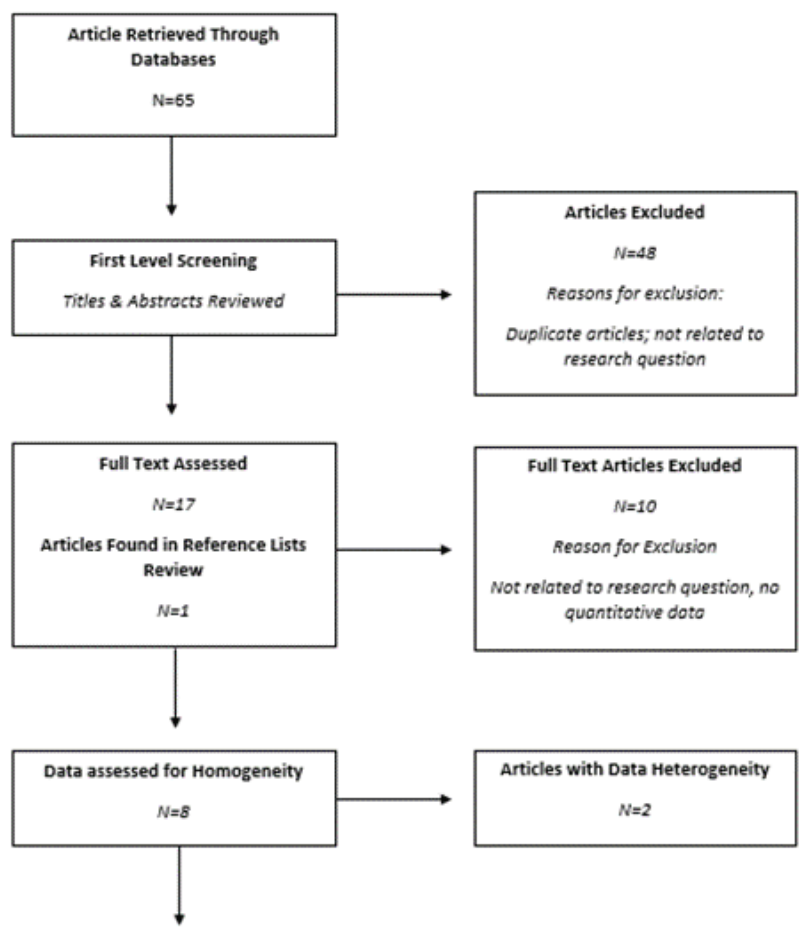

Articles Included in Review $N=6$

Figure 1. - Flow diagram outlining the systematic review and reasons for article exclusion 
inclusion criteria and were determined appropriate for the meta-analysis. On reviewing the data, 2 articles failed to share common outcomes with the remaining articles which prevented meta-analysis. They were removed leaving a total of 6 articles to be used in the meta-analysis.

\section{Primary Outcomes}

The first primary outcome was Time-To-Surgery. A total of 5 articles reported on admission to operation interval. While Franklin et al. (2018) (5), Rutenberga et al. (2018) (6) and Schermann et al. (2019) (7) reported mean and SD ; Mullens et al. (2018) (10) and Tran et al. (2015) (14) used median and IQR. To compensate for this, the median was substituted as mean and the IQR were converted to $\mathrm{SD}$ by calculating IQR/1.35 (12). Furthermore, Schermann et al. (2019) (7) data was reported in two treatment specific categories, hemiarthroplasty and closed reduction internal fixation. This data was included as two entries in figure 2. Another article, Bruckbauer et al. (2019) (4), reported on time to surgery for the DOAC group but did not for the control group and was excluded. There was a significant difference in the time it took from admission to surgery with a mean difference of 14.52 hours (CI 95\% 4.27hrs-24.76hrs). This is expected since current recommendations (15) suggest delaying operative management in patients who take a DOAC.

The other primary outcome was all-cause mortality at 12 months post operation which was reported by three studies. In this data series, there was no statistical difference between the experimental group (DOAC users) and the control group $(\mathrm{RR}=$ 1.06, CI : $0.78-1.44$ ).

In addition to this finding, the 1 month postoperative all-cause mortality also reported no statistically significant difference between the experimental (DOAC) group compared to control group (RR : $0.60 \mathrm{CI}: 0.16-2.22$ ). Franklin et al. (2018) (5) reported two extra patients at 1 month mortality compared to 12 month mortality as these patients where followed up 'as required' and subsequently were excluded.

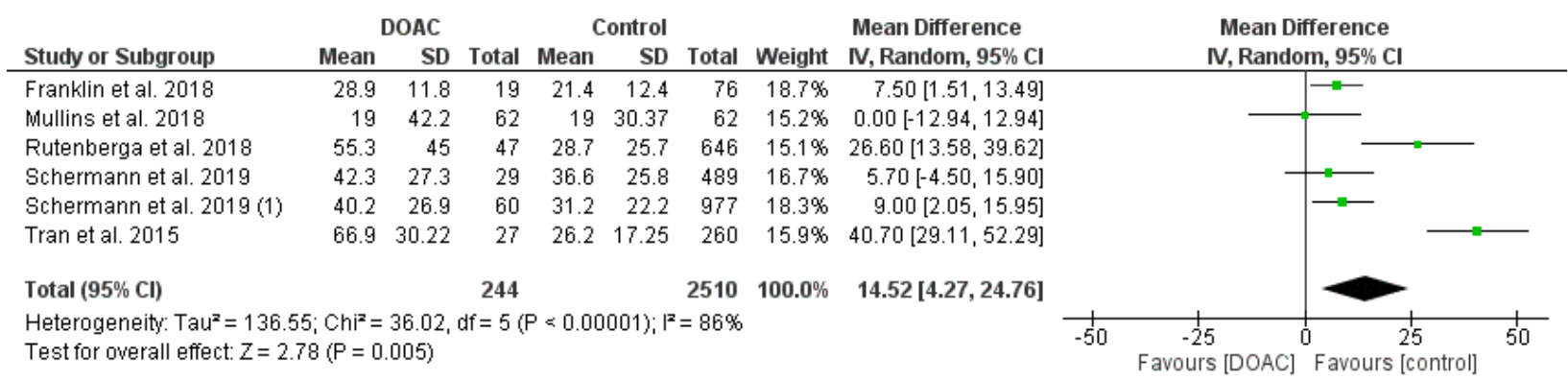

Figure 2. - Forrest-plot of difference in mean time to surgery (hours) between anti-coagulated patients and control.

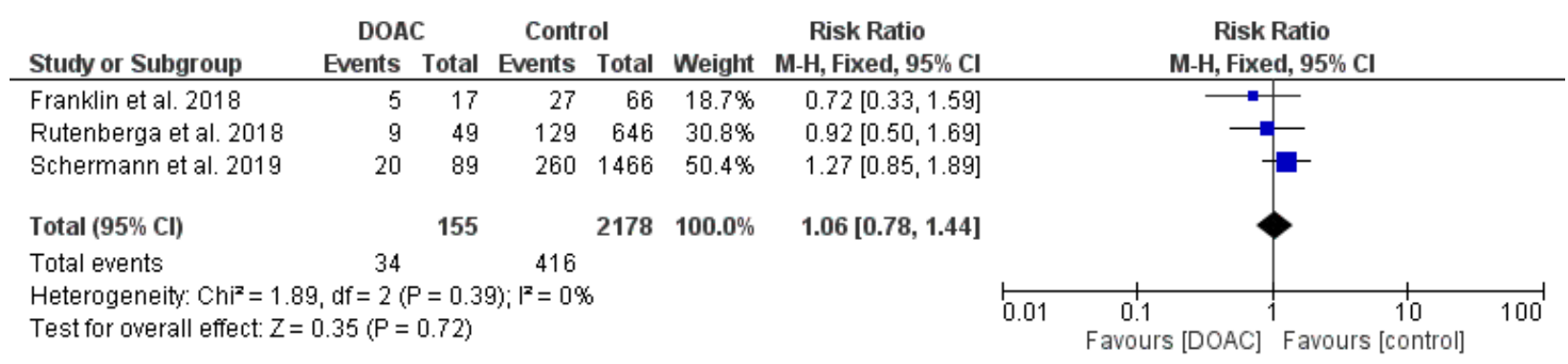

Figure 3. - Forrest-plot of 12 month mortality between anti-coagulated patients and control. 


\begin{tabular}{|c|c|c|c|c|c|c|c|c|c|c|}
\hline Study or Subgroup & \multicolumn{2}{|c|}{ DOAC } & \multicolumn{2}{|c|}{ Control } & Weight & $\begin{array}{c}\text { Risk Ratio } \\
\text { M-H, Random, } 95 \% \mathrm{Cl}\end{array}$ & \multicolumn{4}{|c|}{$\begin{array}{c}\text { Risk Ratio } \\
\text { M-H, Random, } 95 \% \mathrm{Cl}\end{array}$} \\
\hline Franklin et al. 2018 & 1 & 19 & 12 & 76 & $25.7 \%$ & $0.33[0.05,2.41]$ & & $\square$ & & \\
\hline Mullins et al. 2018 & 1 & 62 & 5 & 62 & $23.7 \%$ & $0.20[0.02,1.66]$ & & & - & \\
\hline Schermann et al. 2019 & 6 & 89 & 73 & 1466 & $50.6 \%$ & $1.35[0.61,3.03]$ & & & - & \\
\hline Total $(95 \% \mathrm{Cl})$ & & 170 & & 1604 & $100.0 \%$ & $0.60[0.16,2.22]$ & & & & \\
\hline Total events & 8 & & 90 & & & & & & & \\
\hline $\begin{array}{l}\text { Heterogeneity: } \operatorname{Tau}^{2}=0 \text {. } \\
\text { Test for overall effect: } Z\end{array}$ & $\begin{array}{l}1 ; \mathrm{Chi}^{2}= \\
0.77(\mathrm{P}=\end{array}$ & $\begin{array}{l}4.15, d f \\
0.44)\end{array}$ & $f=2(\mathrm{P}=$ & $0.13) ;$ & $=52 \%$ & & 0.01 & $\begin{array}{c}0.1 \\
\text { Favours [DOAC] }\end{array}$ & $\begin{array}{cc} & 10 \\
\text { Favours [control] }\end{array}$ & 100 \\
\hline
\end{tabular}

Figure 4. - Forrest-plot of 1 month mortality between anti-coagulated patients and control.

\section{Secondary Outcomes}

There was also no statistical difference in rate of perioperative transfusions between the control group compared to the DOAC group with an odds ratio of 1.31 (CI. 0.89-1.92).

Finally, in an analysis of the peri-operative risk of deep vain thrombosis (DVT) development the results were not statistically significant with a risk ratio of 1.25 (CI : 0.15-10.52). Only two articles reported on DVT risk. Tran et al. (2015) (14) was excluded as the article reported pulmonary embolism cases rather than DVT development.

\section{DISCUSSION}

The primary results show that patients who are anti-coagulated on doac had a significantly longer wait to surgery following admission yet this did not increase mortality at both one month or one year post-operatively. This is a particularly interesting finding because the literature is clear that early intervention results in reduced hospital length of stay, morbidity, and mortality (14,16-22). As stated earlier, 2 of the studies $(10,14)$ reported median and IQR which needed to be converted to mean and SD. This was achieved by substituting the median for

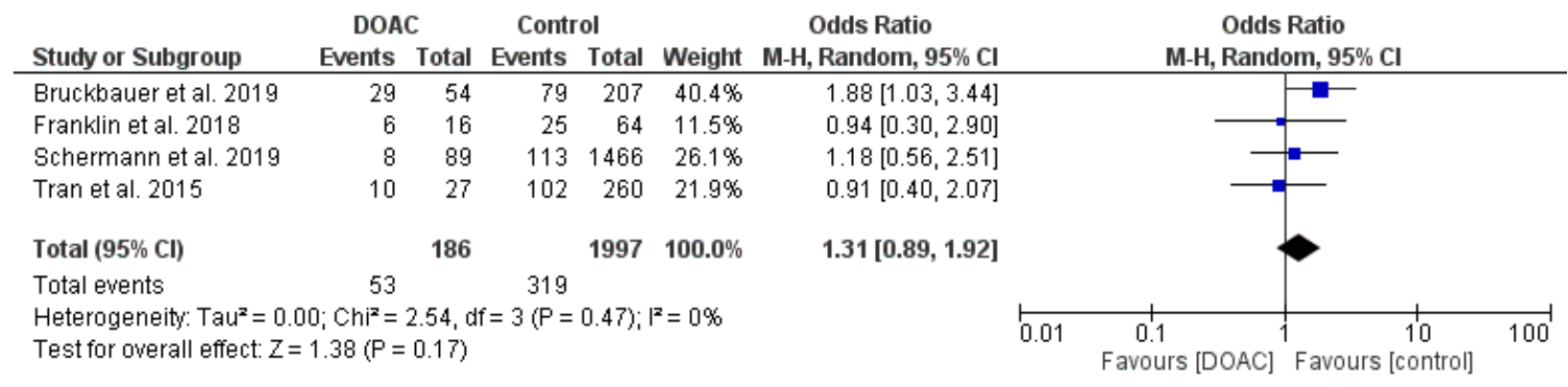

Figure 5. - Forrest-plot of the probability a patient will require a transfusion during the perioperative period.

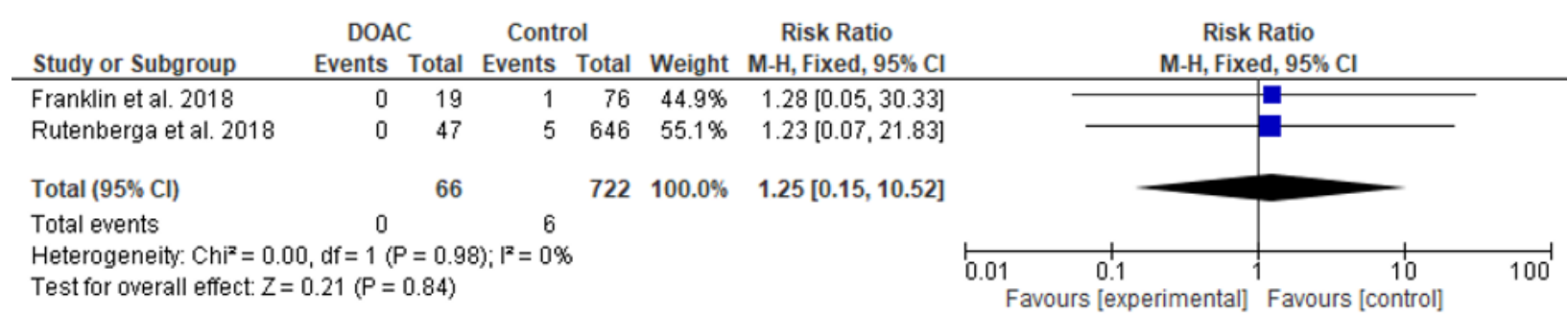

Figure 6. - Forrest-plot assessing the risk of developing a deep vein thrombosis between anti-coagulated patients and control. 
the mean and the IQR/1.35 to give an approximate $\mathrm{SD}$ (13). While this is a well recognised method, accuracy relies on an even distribution of data which is unlikely due to the nature of the topic. Furthermore, as the authors did not report a mean and $\mathrm{SD}$ it suggests that there was an uneven distribution of data. Nevertheless, even with these barriers, there was a statistically significant difference in time-tosurgery between the two cohorts.

The second primary outcome was all-cause mortality which showed a risk ratio of 0.60 at one month post-operatively and 1.06 at one year postoperatively. This is a particularly interesting finding as this cohort had a delay to operative management, yet this delay did not result in a higher mortality rate. While an interesting finding, these two outcomes should be compared cautiously as the data used for the forest plots of figure 2 and figure 3 were derived from different journal articles. Nevertheless, there is a plethora of research which shows improved outcomes with timely operative management (14,16-22); yet, the absence of a mortality benefit for the control group is an interesting finding. Of course, this finding is limited to survivability and no comment is made on other important factors, namely morbidity and hospital length of stay.

We postulate that there are compounding factors which may have resulted in this finding. DOAC require patients to diligently follow a strict medication regime which suggests that these patients may have greater health literacy (23). It is also easy to assume that the control population has less co-morbidities, yet it must be considered that some members of control groups may not be receiving appropriate medical treatment, thus increasing their risk. This oversight could lead to outliers which may have an impact on the results. In comparison, the DOAC population's greater health literacy suggests engagement with medical services. This engagement in rehabilitation services may also play a role in the positive health outcomes as they may be more engaged in post-operative rehabilitation resulting in improved outcomes (2426). Finally, this analysis did not show an increased need for blood transfusions in the DOAC group. This relates to clinical practice as the administration of blood transfusions is often associated with worse outcomes, yet there is controversy regarding a causal relationship (27); nevertheless, the use of DOACs does not increase the rate of peri-operative transfusions.

While this analysis shows these patients do not experience an increase in mortality at one month and one year, this is a developing area of research and literature is consequently lacking. This meta-analysis was limited to six articles, with the secondary outcomes sub-analysis being limited to two to four articles. In addition, the allcause mortality data sets were heavily weighed on Schermann et al. (2019) (7) data. However, there are some take home messages. Based on current data, treating orthopedic surgeons should be reassured that they are not doing a disservice to their patients by delaying surgery in patients who are anticoagulated with DOAC as there is no data to suggest this increases patient mortality. Interestingly, these patients do not require more peri-operative blood transfusions. Unfortunately, there is insufficient evidence to comment on other important health outcomes (length of hospital stay and morbidity) and as such, these will need to be assessed in the future as evidence becomes available.

\section{CONCLUSION}

As the population of patients on long term direct anticoagulation therapy continues to increase, the peri-operative management of hip fractures will increasingly present a management dilemma for the treating team. The key finding is that these patients have a delay to theatre, yet, this does not impact survivability. The treating surgeon should be comforted by these findings as operative management can be safely delayed to reduce the risk of intraoperative bleeding. While research into DOAC has been growing, orthopaedic specific research remains elusive so further research is required to guide best practice.

\section{REFERENCES}

1. Lomas O, Argyle RA, Prendergast BD. Will direct thrombin inhibition change the boundaries of oral anticoagulation?. QJM : An International Journal of Medicine. $2010 ; 103(6): 429-434$. 
2. Charlesworth M, Arya R. Direct oral anticoagulants : perioperative considerations and controversies. Anaesthesia $2018 ; 73: 1460-1463$.

3. Fahim G. Reversal Agents for Direct-Acting Oral Anticoagulants. Drug Topics 2017 ; 161(5) : 36.

4. Bruckbauer M, Prexl O, Voelckel W, Ziegler B, Grottke O, Maegele M. Impact of Direct Oral Anticoagulants in Patients with Hip Fractures. Journal of Orthopaedic Trauma. 2019 ; 33(1) : 8-13.

5. Franklin NA, Hurley RK, Mir HR, Beltran MJ. Outcomes of early surgical intervention in geriatric proximal femur fractures among patients receiving direct oral anticoagulation. Journal of Orthopaedic Trauma. $2018 ; 32: 269-73$.

6. Rutenberg TF, Velkes S, Vitenberg M, Leader A, Halavy Y, Raanani P, Yassin M, Spectre G. Morbidity and mortality after fragility hip fracture surgery in patients receiving vitamin $\mathrm{K}$ antagonists and direct oral anticoagulants. Thrombosis Research. 2018 ; 166 : 106112 .

7. Schermann H, Gurel R, Gold A, Maman E, Dolkart O, Steinberg EL, Chechik O. Safety of urgent hip fracture surgery protocol under influence of direct oral anticoagulation medications. Injury. 2019 ; 50(2) : 398402.

8. Grandone E, Ostuni A, Tiscia GL, Marongiu F, Barcellona D. Management of Patients Taking Oral Anticoagulants Who Need Urgent Surgery for Hip Fracture. Seminars in Thrombosis \& Hemostasis. 2019 ; 45(2) : 16470.

9. Lott A, Haglin J, Belayneh R, Konda SR, Leucht P, Egol KA. Surgical Delay Is Not Warranted for Patients With Hip Fractures Receiving Non-Warfarin Anticoagulants. Orthopedics. 2019 ; 42(3) : 331-335.

10. Mullins B, Akehurst $H$, Slattery $D$, Chesser $T$. Should surgery be delayed in patients taking direct oral anticoagulants who suffer a hip fracture? A retrospective, case-controlled observational study at a UK major trauma centre. BMJ open. $2018 ; 8(4)$ : e020625.

11. Yassa R, Khalfaoui MY, Hujazi I, Sevenoaks H, Dunkow P. Management of anticoagulation in hip fractures: A pragmatic approach. EFORT Open Rev. 2017 ; 2(9) : 394402.

12. Herbolsheimer P, Swain SM. Meta-analysis : Should It be More Than the Sum of Its Parts?, JNCI : Journal of the National Cancer Institute, 2010 ; 102(24) : 1817-1819.

13. Higgins JPT, Thomas J, Chandler J, Cumpston M, Li T, Page MJ, Welch VA (editors). Cochrane Handbook for Systematic Reviews of Interventions version 6.0 (updated July 2019). Cochrane, 2019. Available from www.training. cochrane.org/handbook.

14. Tran T, Delluc A, De Wit C, Petrcich W, Le Gal G, Carrier M. The impact of oral anticoagulation on time to surgery in patients hospitalized with hip fracture. Thrombosis Research. 2015 ; 136(5) : 962-965.
15. Website: Clinical Excellence Commission 2017. Nonvitamin K Antagonist Oral Anticoagulant (NOAC) Guidelines. Available at: http://www.cec.health.nsw.gov.au/ data/assets/pdf file/0007/326419/noac guidelines.pdf. Accessed December 12, 2019.

16. Eardley WG, Macleod KE, Freeman H, Tate A. "Tiers of delay" : warfarin, hip fractures, and target-driven care. Geriatr. Orthop. Surg. Rehabil. 2014 ; 5(3) : 103-108.

17. Elliott J, Beringer T, Kee F, Marsh D, Willis C, Stevenson M. Predicting survival after treatment for fracture of the proximal femur and the effect of delays to surgery. J. Clin. Epidemiol. 2003 ; 56 : 788-795.

18. Ftouh S, Morga A, Swift C. Management of hip fracture in adults : summary of NICE guidance, BMJ. $2011 ; 342$ : d3304.

19. Khan SK, Kalra S, Khanna A, Thiruvengada MM, Parker MJ. Timing of surgery for hip fractures : a systematic review of 52 published studies involving 291, 413 patients. Injury. $2009 ; 40(7)$ : 692-697.

20. Leung F, Lau TW, Kwan K, Chow SP, Kung AW. Does timing of surgery matter in fragility hip fractures? Osteoporos. Int. $2010 ; 21$ : 529-534.

21. Roberts KC, Brox WT, Jevsevar DS, Sevarino K. Management of hip fractures in the elderly. J. Am. Acad. Orthop. Surg. $2015 ; 23: 131-137$.

22. Ryan DJ, Yoshihara H, Yoneoka D, Egol KA, Zuckerman JD. Delay in hip fracture surgery: an analysis of patient-specific and hospital-specific risk factors. J.Orthop. Trauma. $2015 ; 29(8)$ : 343-348.

23. Yiu A, Bajorek B. Patient-focused interventions to support vulnerable people using oral anticoagulants : a narrative review. Ther Adv Drug Saf. 2019 ; 10 : 2042098619847423.

24. Galea MP, Levinger P, Lythgo N, Cimoli C, Weller R, Tully E, McMeeken J, Westh R. A targeted home-and center-based exercise program for people after total hip replacement : a randomized clinical trial. Archives of physical medicine and rehabilitation. 2008 ; 89(8) : 1442-7.

25. Robertson NB, Warganich T, Ghazarossian J, Khatod M. Implementation of an accelerated rehabilitation protocol for total joint arthroplasty in the managed care setting: the experience of one institution. Advances in Orthopedic Surgery. $2015 ; 29$ : 1(7).

26. Stockton KA, Mengersen KA. Effect of multiple physiotherapy sessions on functional outcomes in the initial postoperative period after primary total hip replacement : a randomized controlled trial. Archives of physical medicine and rehabilitation. 2009 ; 90(10) : 1652-1657.

27. Danninger T, Rasul R, Poeran J, Stundner O, Mazumdar M, Fleischut PM, Poultsides L, Memtsoudis SG. Blood Transfusions in Total Hip and Knee Arthroplasty: An Analysis of Outcomes. The Scientific World Journal. $2014 ; 623460: 1-10$. 